\title{
APPROACH FOR VISUALIZATION OF UNCERTAINTY IN CAD-SYSTEMS BASED ON ONTOLOGIES
}

\author{
Lucia Mosch \\ Technische Universität Darmstadt \\ Department of Computer \\ Integrated Design \\ Darmstadt, Germany \\ I.mosch@dik.tu-darmstadt.de
}

\author{
André Sprenger \\ Technische Universität Darmstadt \\ Department of Computer \\ Integrated Design \\ Darmstadt, Germany \\ sprenger@dik.tu-darmstadt.de
}

\author{
Reiner Anderl \\ Technische Universität Darmstadt \\ Department of Computer \\ Integrated Design \\ Darmstadt, Germany \\ anderl@dik.tu-darmstadt.de
}

\begin{abstract}
In this paper an approach for controlling uncertainties in load-carrying systems in virtual product development during the phase of product design will be presented. In design, manufacturing and usage of technical products uncertainties arise according to process properties and they influence products properties. Many of these properties impact each other. These facts lead to deviation of expected property values which are shown for example in the approximation of stress and strength. In cases of load carrying systems misjudgment can lead to disastrous consequences. In this paper an approach for the visualization of information about uncertainty in loadcarrying systems in CAD (Computer Aided Design)-systems within the Collaborative Research Center (CRC) 805: "Control of Uncertainty in Load-Carrying Systems in Mechanical Engineering" funded by the DFG (Deutsche Forschungsgemeinschaft) will be described. The representation of the properties' interdependency is to be expressed by an ontology based system. The visualization of the properties and the information about uncertainty is demonstrated in a CADsystem.
\end{abstract}

\section{INTRODUCTION}

In the product lifecycle phases design, manufacturing and usage of technical products uncertainties occur according to process properties and they impact products properties. Many of these properties have an influence on each other. In the product design process many property values have to be fixed and their influence on the product behavior and their interaction have to be considered. Based on an information model considering ontology, clarifying the influence of properties of the product as well as the visualization of this information based on a geometry model in CAD-systems can be realized during the early phases of product development.

The goal of the approach is to allow engineers to control and reduce uncertainty through a visualization of product properties and their influence on each other in product design.

One requirement for product modeling using CAD-systems is the estimation of prospective properties. The estimations, however, are of different quality. These differences in estimations result e.g. in oversizing the developed product using a factor of safety. To visualize the dependent properties and their consequences for product development is the core of the described approach aiming at an increase of estimation quality and a reduction of uncertainty. It is essential to detect uncertainties and their correlation in order to control them. The representation of these detected uncertainties and their correlation are based on ontologies. The aim of this approach is to link these uncertainty related information which is deposited in a database with the CAD model and to visualize uncertainties on the geometric model. Therefore the long-term objective is to increase the efficiency for the designer combined with a reduction of product oversizing enabling however robust design.

\section{CURRENT SITUATION}

The product life cycle can be subdivided into three phases:

- Virtual product creation,

- manufacturing and

- usage with recycling. 
Virtual product creation covers the processes of product development and production planning. Product development in turn can be described with the phases of

- product planning,

- product conception,

- preliminary product design and

- product detailing.

The focus in this paper is on preliminary product design, in particular the visualization of uncertainty within this phase. Product design is supported by modern virtual technologies. In this context CAx-technologies, in particular CAD-systems, have reached a high application range in the last decades [1]. Due to this trend, geometrical modeling was expanded by virtual technologies for analysis, simulation and optimization until start of production (SOP). The results of the usage of virtual technologies are virtual products [2].

CAD-systems represent the products in a hierarchical structure called model structure. It provides a hierarchical aggregation of components which describe a product. With the model structure, the understanding of the components which describe the product as well as the properties of the components can be represented. The components described in the model structure are assemblies and subassemblies as well as parts [3], material, features, parameters and constrains.

Another aspect regarding virtual product creation illuminates management methods. Management methods are targed-oriented methods and tools, which facilitate product development to become more efficient from an integrated and global viewpoint [4]. Management methods such as simultaneous engineering (SE) support a concurrent development of products and production processes [5]. SE is a workflow controlled simultaneous development process that passes through a number of operations in parallel instead of working sequentially (Figure 1). SE integrates the aims of product development with high quality, shorter time and costs.

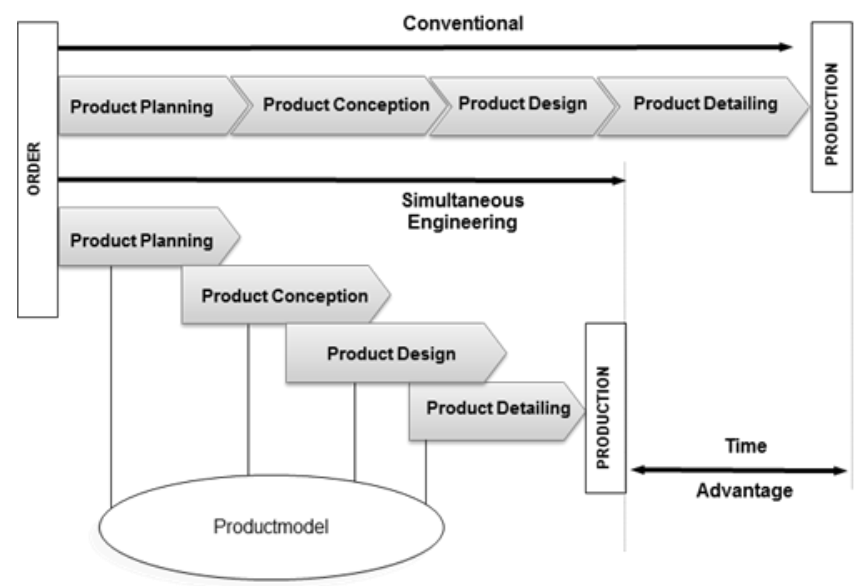

Figure 1: Simultaneous engineering of processes [6]

In the product development process requirements have to be arranged and future properties of the substantial product have to be identified and property values have to be astimated.
By this estimation uncertainty occurs [7]. The topic of uncertainty has been discussed in the scientific field from a variety points of view e.g. data uncertainty, measurement uncertainty, uncertainty of information and many more. In technical literature, there are numerous definitions of uncertainty. Uncertainty is often associated with safety or risk [8]. In the field of economics uncertainty is understood as a state in which a deliberately perceived lack of safety exists. Nevertheless, a lack of safety is also often equated with danger. [8]. Safety is a situation, where risk is smaller than the limiting risk [9].

The CRC 805: "Control of Uncertainties in Load-Carrying Structures in Mechanical Engineering" develops new approaches for the control of uncertainties in load carrying structures. The CRC developed a working hypothesis and a model for the classification of uncertainties:

"Uncertainty occurs when process properties of a system cannot be determined. Uncertainty can be described and can be quantified with known methods of risk-analysis. " [7]

Thereby uncertainties are created in processes by the system which is involved in the process. This system enfolds all relevant properties which are necessary for the determination of the output of the process which can be described as outputstate. The effect of uncertainty is visible in the scattering of output states of a certain process with seemingly equal input parameters.

In order to determine process properties information about the system is necessary. The model of uncertainty shown in Figure 2 classifies the available information in three classes. Ignorance describes the lack of information and missing awareness. With incertitude the effect on the process is known and could be quantified partially.

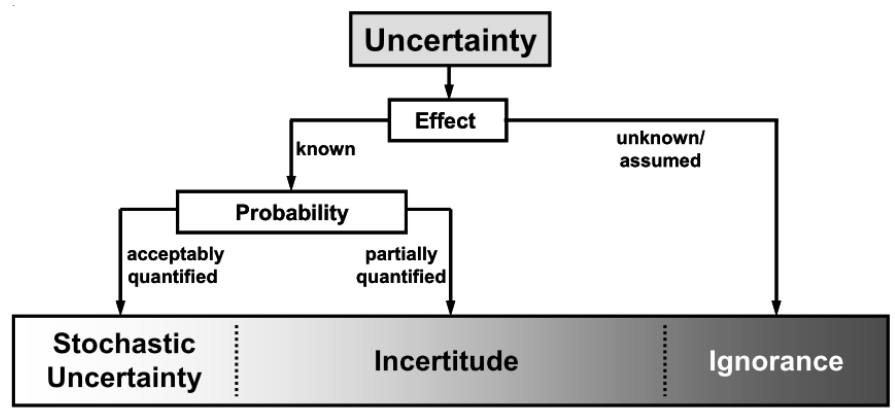

Figure 2: Model of uncertainty [10]

In the case of stochastic uncertainty widespread information about the system and properties which determine the output is available. Relating to concrete values the model describes different stages of information. 


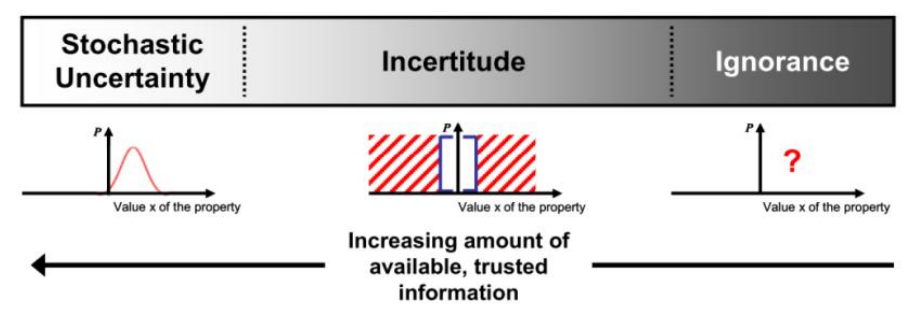

Figure 3: Categories of uncertainty [10]

As shown in Figure 3 ignorance has no concrete value; for incertitude there is a range of possible values given; in case of stochastic uncertainty the distribution function is given. With the increasing amount of information about properties and the effects on the system uncertainty can be reduced. In product design the characteristics and properties of the product are engineered. For designing products which meet the requirements not by oversizing uncertainty has to be avoided.

The amount and quality of available information is a critical factor for the control of uncertainty [11, 12]. In this context it means: more information content reduces uncertainty.

Therefore, in product development the necessity for optimal control of uncertainties by a visualization of individual uncertainties in the appropriate context does exist. During the phase of product design the importance of getting information about the possible impacts and correlations of any preferred properties increases. With this information the product can be controlled from the beginning of the early phases of product development. In this context target oriented provision of information results in reduction of uncertainty. The challenge is to orchestra information of all phases of the product life cycle (virtual product creation, manufacturing, usage) in terms of SE to ensure an effective and efficient workflow in the product design. The efficient reuse of information requires the support of a structured information model and an accessible presentation of information (Figure 4).

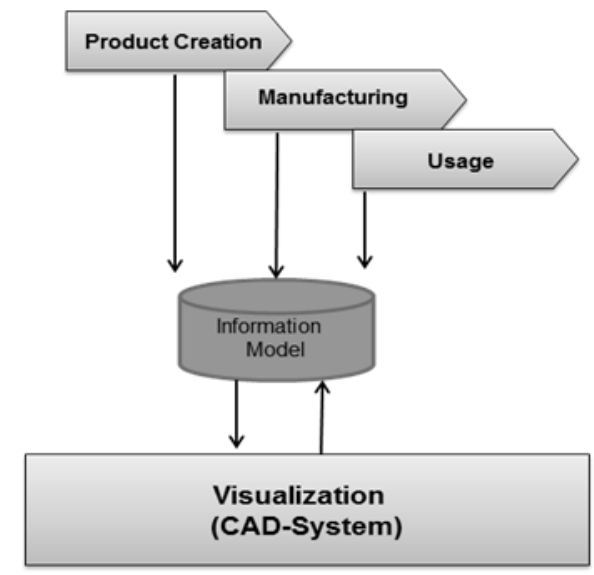

Figure 4: Product life cycle in terms of SE

Ontologies are considered as an appropriate method to be used for classification of information [13].

\section{REQUIREMENTS}

The aim of the present work is to develop an approach to link information about uncertainties with a CAD model and to visualize uncertainties. Information about uncertainty result from an ontology based model. Within this work a known and given ontology is assumed. This approach is focused on visualization of the information within the phase of product design. The known information about the single uncertainties should be visualized within a CAD-system directly for a given CAD-model.

After introducing the current situation, the description of the requirements for the visualization of information about uncertainty in a CAD-system will be given. The core requirement for the visualization of uncertainties is the independent and neutral presentation of these uncertainties (Figure 5). This presentation results from representation of the model structure as well as the related and linked information. The representation of the model structure is described in a CAD-system; the representation of related linked information is a result of the ontology.

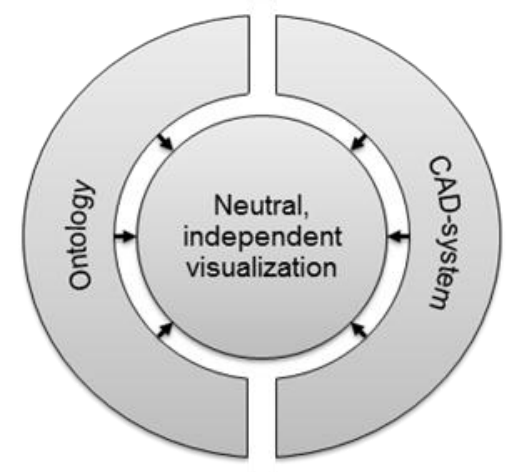

Figure 5: Core requirement for the approach

Regarding to the core requirement, several further requirements have to be fulfilled. These further requirements have been identified:

- Knowledge and experience about the respective technical product is necessary: For the management and visualization of information knowledge and experience have to be collected.

- System-independence has to be ensured: A product design process is a collection of processes with different areas of expertise, expert knowledge and usage of various engineering tools like CAD-systems.

- Interfaces between the domain ontology and the domain visualization of the represented model structure have to be identified. The model structure has to be interpreted and linked to the ontology. The model structure has to express properties.

- The merging of information about uncertainty and appending properties has to be presented in a format, the user can interact with. The usability has to be ensured through a graphical user interface (GUI). 
- The user only has to use the information presented in the GUI (front-end). Knowledge about the representation within the ontology should be encapsulated and only be accessible for the expert (back-end).

- A customized view of data is needed: To avoid misunderstandings in the presentation of information and to guarantee the clarity, not all information appearing in the respective property can be visualized at the same time. Hence, it is necessary to limit the information. The limitation can be realized through many points of view, e.g. which kind of information, in which phase of product life the uncertainty occurs, etc. To meet this requirement, single uncertainties must be listed as a structure. Besides, the possibility of sorting the uncertainties must be given.

- For the purpose of SE all information about uncertainty along virtual product creation, manufacturing and usage has to be integrated in parallel.

\section{APPROACH}

The aim of the approach of visualization of uncertainties based on ontologies creates a possibility to capture, save, categorize and allocate uncertainties during the product design process. The focus of the approach is to integrate information about uncertainties and its correlations from already existing databases into a CAD-system.

The efficient reuse of knowledge in product design requires the support of an information based model. The representation of the interdependencies of properties has to be supported by an ontology based system. The properties and the information about uncertainty should be linked with a CADsystem. These properties and the information should be described system-independent. The aim of the approach is to couple information about uncertainty to the geometry model. This information is managed in an ontology based model, where the information is linked with the properties. The building of coherences between information and properties will be solved by an ontology tool. The visualization of the information linked with the geometric model will be implemented within a CAD-system.

The approach is divided into three parts (Figure 6):

1. Ontology,
2. CAD-system and

3. Information management (browser) connected with ontology and CAD-system.

Furthermore the connection between these three parts has to be implemented by standard interfaces. As standard interfaces OWL (Web Ontology Language) API (Application Programming Interface) and CAD-specific API seem to be appropriate.

For uncertainties the cause and effect relations of different processes and their outcome states is very important. Outcome states are input states for successive processes. These cause and effect relations tend to become very complex. Thus ontologies are chosen for the representation and the processing of uncertainty related information.

Common supporting tools in virtual product design are CAD-systems. During the process of design CAD-systems are currently used to support design engineers. Typical geometric models support capturing parametric information and geometric constraints of components. Information about uncertainty is not included nor supported. The use of a CAD model and the knowledge about uncertainty could make the use of CADsystems much more efficient and effective for designer.

Information management (browser) builds upon the representation of data model in a runtime environment and acts as a platform-independent visualization of the information considering ontology and the CAD-system. An essential basic condition of the browser within the runtime environment is to act as an "uncertainty database", characterized as a browser to manage uncertainty information. This database must contain all available information with regard to single uncertainties as well as their individual effects. Influenced information and their effects are listed in the browser in a tabular presentation. Besides, different algorithms have to be implemented which limit and filter information by use of a view-concept. The possibility of making decisions between view of an e.g. assembly or individual part is decided by the customer. On the one hand the correlations between all information are managed in an ontology based model, on the other hand the information related to the modeled product (model structure / product structure) results from the CAD-system.

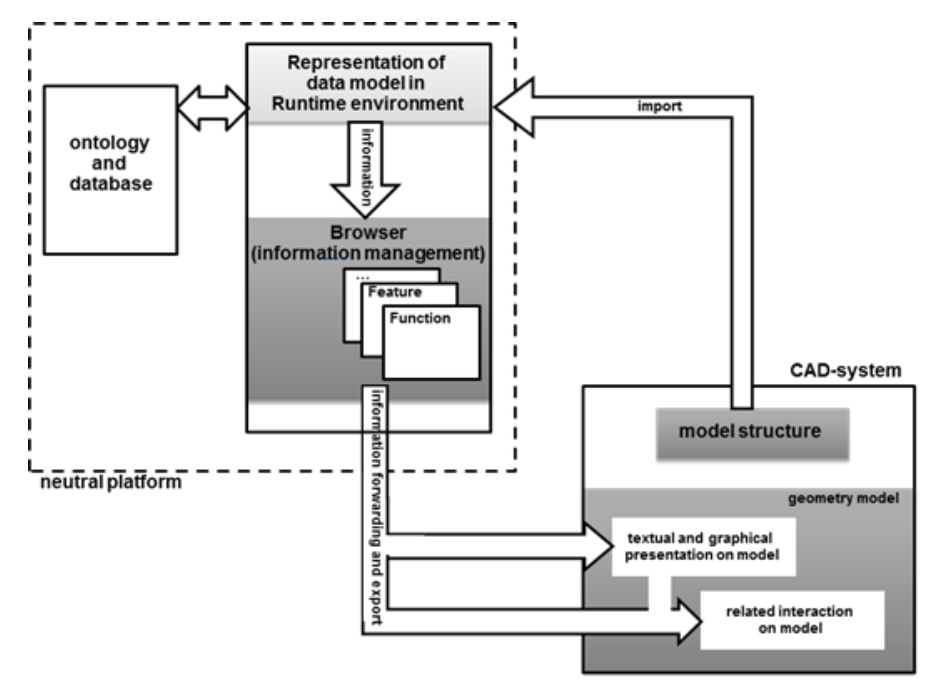

Figure 6: Approach including connection between ontology and CAD-system

To visualize information about uncertainty in a CAD model, the usage of an interface to the CAD-system is necessary. A CAD-specific API must be used. Information like model structure is essential to be accessed, interpreted and imported into the runtime environment. Within a CAD-system technical products are composed of assemblies, subassemblies and parts. Furthermore subassemblies or parts consist of 
features, to those properties are suspended. This is the basis for the linking of information about uncertainty and the CAD model. After importing the model structure with its properties into the runtime environment, a query to the ontology will be sent. All answers of the queried information will be matched to the properties of the model structure. In response to the correlation between the requested information and the properties of the model structure, the combined information will be exported into the browser. After this process, selected information will be presented directly textually or graphical on the related CAD model and related property. The user, in this fact the engineer or designer, will be supported in his work. Furthermore, there will be the potential to interact with and manipulate the model. Consequently the concept of the bidirectional associativity of parametric CAD-systems will be supported. Parametric modifications of the geometric model as well as the usage of CAE-technologies like Finite Element Analyses (FEA) will be possible. The interaction and manipulation of the geometric model with the browser is topic of further phases of the project.

\section{CURRENT WORK}

After describing the approach for visualization of information about uncertainty based on ontology, the current browser for linking the geometric model with external information collected in the browser will be explained. The browser is the designed connection area between the ontology editor and the CAD-system. The function of this browser is to categorize the information with the using the view-concept. Figure 7 shows the GUI of the implemented browser for the management of inked information. Information is presented in a tabular form and structured by a choice of a certain view.

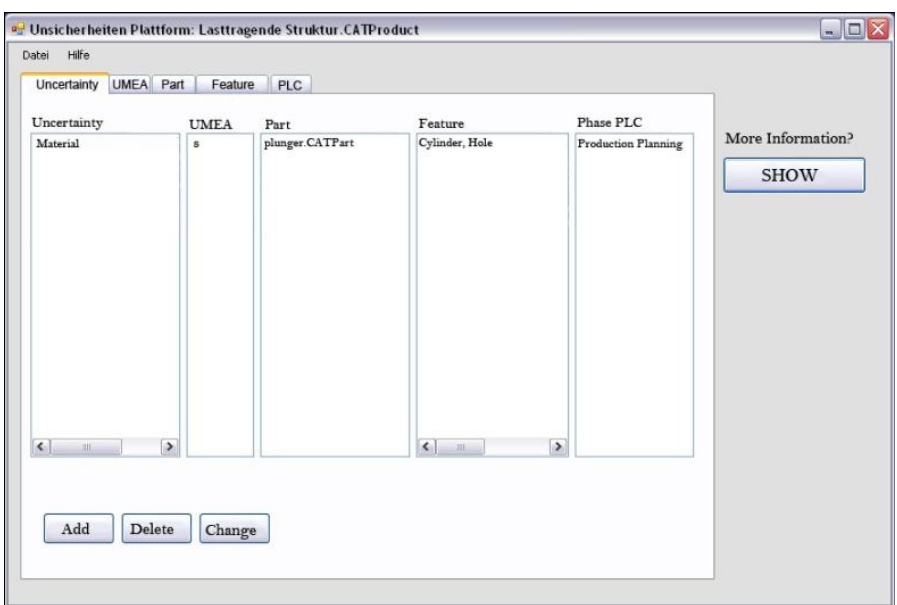

Figure 7: The browser unit

In this early phase of our work the user has to connect and manage information manual. This means the correlation between the geometry model and the information about uncertainty results from manually selection. For the creation of the links an input bar is implemented in the module. The connection is implemented using the model structure. The model structure contains the assemblies and individual parts, as well as the single features used in the model. Therefore the model structure from the CAD-system has to be accessed and imported into the input bar to the various fields (Figure 8).

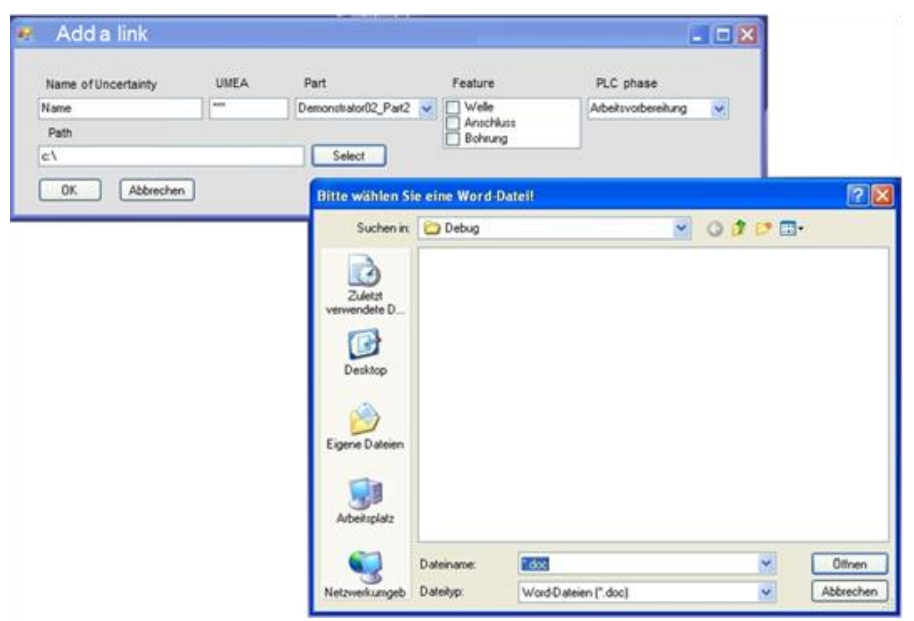

Figure 8: A bar to add and connect information

The user has to name the type of uncertainty, connect information about uncertainty (resulting from an external document from a database) along the hierarchy of the model with the assembly, part, feature or property and select the phase in which the uncertainty occurs. By the manual linking of information within the browser a semantic connection will be simulated. After confirming the entry correlated data will be imported into the browser. Besides the creation of links also the removal and changing of existing links is an important part of the browser. The removal and changing is also implemented within the same input bar.

In addition to the management of data within the browser, the import and export of connected data in a neutral data format is an important part of the functionality of the presented approach. For the preliminary work the neutral *.dat-format was chosen. It is a common data format which is used by various applications. In addition, files in *.dat-format can be opened and changed by various editors without using the applications the files were created from.

To improve the handling and overview of the information, a view bar is implemented in the browser unit. This bar allows the user to limit the presentation of information and their links. In this phase the implemented categories are:

- short name of the uncertainty,

- part and feature the uncertainty belongs. and the

- phase (PLC - product life cycle phase) in which the uncertainty occurs.

After selecting a bar, the information will be arranged along the selected category (Figure 9). 


\begin{tabular}{|l|l|l|l|l|}
\hline Uncertainty & UMEA & Part & Feature & PLC \\
& & & \\
Uncertainty & UMEA & Part & Feature \\
\hline Material & 8 & plunger.CATPart & Cylinder, Hole \\
\hline
\end{tabular}

Figure 9: View concept - customized view of the data

The imported and linked information is presented in the browser in a tabular form. It acts primarily for considering and capturing the information and its correlations. However, the selection of individual information results as a visualization of linked information directly on the CAD model. Today, product manufacturing information (PMI) is state of the art to visualize manufacturing annotation. The visualization of linked information of uncertainty should be realized in an similar way. The visualization of uncertainty within the CAD-system is based on the import of information resulting from a database in the background to the corresponding geometric model. This procedure is associated with a high computational effort. To avoid unwanted computational effort, the choice of individual uncertainties is confirmed before visualization. This concept is implemented by the "SHOW"-button within the browser (Figure 7).

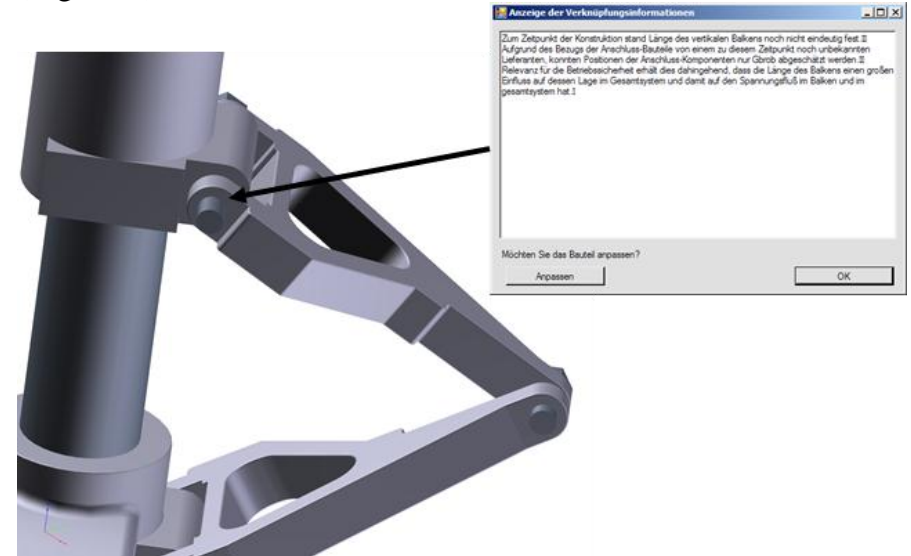

Figure 10: presentation of uncertain related information on the geometric model

According to the presented approach visualization is attached to the CAD-system in the geometric model. In this early phase of the implementation the presentation of the information about uncertainty is done by a textual annotation (Figure 10). For the text-based presentation, a separate interface within the CAD-system is implemented where selected information will be imported.

\section{FUTURE WORK}

A necessary step to master uncertainty is the collection of information about the technical product and the cause and effect of uncertainty. The ontology in combination with a database serves as storage for uncertainty related information. The role of the ontology model is to link information with different types of relations. This information is categorized by the ontology in order to present the interdependencies of different aspects and the according information in the browser.
A further development of the visualization is to accent information on a geometric model within a CAD-system. By a colored accentuation on the geometric model the text-based information is clear and intuitive for the user.

Within the approach an interaction with the geometric model is provided. In this context a modification and manipulation of geometry should be possible after recieving the information, e.g. that one property is over dimensioned.

Other methods, like Finite Element Analysis, should be integrated. This is part of prospective research and development activities.

\section{ACKNOWLEDGMENTS}

We would like to thank the Deutsche Forschungsgemeinschaft (DFG) for funding this project within the Collaborative Research Centre (CRC) 805. The development of an ontology based information model for uncertainty is part of the subproject A5 "Informationsmodell zur Repraesentation und Visualisierung von Unsicherheiten" (Data Model for the Representation and Visualization of Uncertainties) of the Collaborative Research Centre 805 "Beherrschung von Unsicherheit in lasttragenden Systemen des Maschinenbaus" (Control of Uncertainty in Load-Carrying Structures in Mechanical Engineering) funded by Deutsche Forschungsgemeinschaft (DFG, German Research Foundation). For further information visit www.sfb805.tu-darmstadt.de.

\section{REFERENCES}

[1] Krause, F.-L., Franke, H.-J., Gausemeier, J.: Innovationspotentiale in der Produktentwicklung, HanserVerlag, München, Wien, 2006

[2] Spur G.; Krause, F.-L.: Das virtuelle Produkt Management der CAD-Technik. Carl-Hanser-Verlag, München, Wien 1997

[3] Boothroyd, G.; Dewhurst, P.; Knight, W.: Product Design for manufacturing and assembly, Marcel Dekker AG, 2002.

[4] Eigner, et al.: Engineering Database, München: Carl Hanser Verlag, 1991

[5] Ehrelenspiel, K.: Integrierte Produktentwicklung Denkabläufe, Methodeneinsatz, Zusammenarbeit 4. Auflage, Hanser Verlag, 2009

[6] Anderl, R.: Future in VR and CVE, for Design, Engineering \& Manufacturing, Euro VR-EVE 2010 in Paris/France

[7] Koenen, J. F.; Platz, R.; Hanselka, H.: An Approach to Quantify the Influence of Uncertainties in Model-based UsageMonitoring of Load-Carrying Systems: ISMA 2010 International Conference of Noise and Vibration Engineering, 20. - 22.09.2010 in Leuven 
[8] Wiese, H.: Mikroökonomik, 4. Auflage, Berlin: SpringerVerlag, 2005

[9] DIN EN 1050: Sicherheit von Maschinen, Leitsätzen zur Risikobeurteilung, 1996

[10] Engelhardt, R. A.; Koenen, J. F.; Enss, G. C.; Sichau, A.; Platz, R.; Kloberdanz, H.; Birkhofer, H.; Hanselka, H.: A Model to Categorise Uncertainty in Load-Carrying Systems: MMEP Modelling and Management of Engineering Processes, 19. 20.07.2010 in Cambridge/UK
[11] Bertsche, B., Lechner, G.: Zuverlässigkeit im Fahrzeugund Maschinenbau - Ermittlung von Bauteil und SystemZuverlässigkeiten, 3. Auflage, Springer-Verlag, 2004

[12] Knetsch, T.: Unsicherheiten in Ingenieurberechnungen, Magdeburg, Univ., Diss., 2004

[13] Thel, M.: Wissensstrukturierung und -repräsentation im Produktentwicklungsprozess, Shaker Verlag, Aachen, 2007 